\title{
Analysis of Energy and Mobility Model on Opportunistic Routing Algorithms
}

\author{
Jiradett Kerdsri \\ Defence Technology Institute \\ Ministry of Defense, Thailand \\ Email: jiradett.k@dti.or.th
}

\author{
Tawiwat Veeraklaew \\ Defence Technology Institute \\ Ministry of Defense, Thailand \\ Email: tawiwat.v@dti.or.th
}

\begin{abstract}
An Opportunistic Network is an extreme network where the communication mainly depends on the mobility of the participating nodes to address the intermittent connectivity. This opportunistic routing utilizes a store-carry-forward paradigm exploiting node mobility and local forwarding to transfer the messages. The mobile nodes are normally low-power portable wireless communication devices employing as wireless ad hoc network for multi-hop transmission. With the limited resource, the performance of the routing protocols can be degraded. Thus, it is vital to consider the energy consumption of the mobile node in this opportunistic environment, especially with different mobility patterns. This paper aims to study and analyze the energy usage on the node mobility. The results from the simulations show that MapBased mobility models consume more energy than random based mobility models while gaining higher delivery ratio. In the random based models, random direction consume more power than random waypoint and random walk respectively.
\end{abstract}

\section{INTRODUCTION}

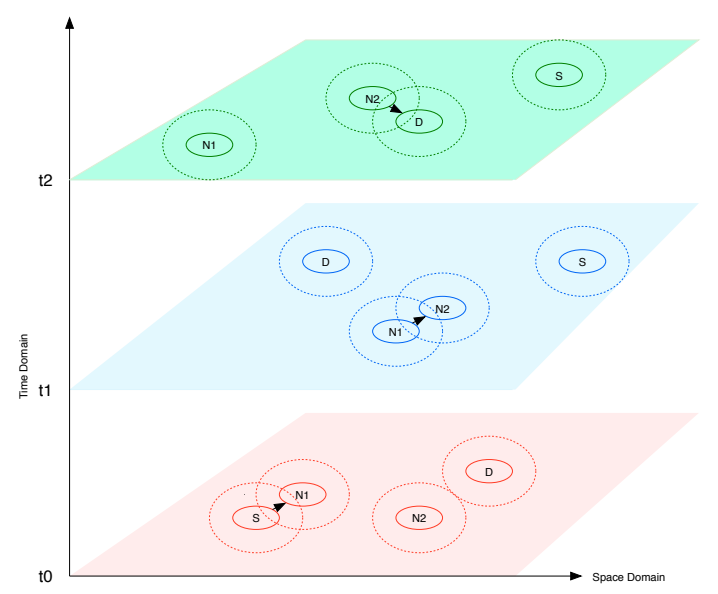

Fig. 1. Store-Carry-Forward routing model

Commonly, an Opportunistic Network (OppNet) is a challenged network consists of the following characteristics [1]:

- Network contacts are intermittent

- An end-to-end path between the source and destination infrequently exists

- Link performance is highly variable or extreme

Therefore, routing in the opportunistic environment requires collaboration among mobile nodes. Formally, opportunistic routing use store-carry-forward (SCF) paradigm to route the messages which any possible node can opportunistically be used as a next hop in order to bring the messages closer to the destination [1]. The example in Fig. 1 shows the SCF model when source node $(S)$ wants to send a message to destination node $(D)$ but there is no connection between them. At time $t_{0}, S$ node contacts node $N_{1}$ and send the messages to $N_{1}$. Node $N_{1}$ has to store the messages and carries them while moving. Then, node $N_{1}$ meets node $N_{2}$ at time $t_{1}$ and sends the messages to node $N_{2}$. Node $N_{2}$ carries the messages and meets node $D$ at $t_{2}$, then the message are sent to the destination node.

A crucial aspect of an opportunistic algorithm evaluation is the mobility pattern of the nodes in order to determine a contact duration as well as a contact pattern. In fact, the mobility pattern can be influenced by physical factors such as obstacles, the speed limits and boundaries defined by the streets and social factors [2]. Even though, mobility is one of the major issues in OppNet research, it can only be studied and not engineered, unlike other important aspects [3].

This paper aims to study the behavior of node mobility models on the energy consumption which is another critical factors on OppNets. In fact, the energy efficiency has long been the main concern in the implementation of routing protocols for MANET to prolong the operational time of network [4], [5]. Since most of the mobile devices in OppNets environment are usually equipped with energy limited battery, the energy efficient protocols are obligation.

The rest of the paper is organized as follows. In Section 2 , we discuss the literature reviews of OppNet routing and existing works. The detail of evaluation is elaborated in Section 3 , which presents the metrics and the result of our simulation. We conclude the paper and point out some future research directions in Section 4.

\section{LITERATURES REVIEW}

In OppNet, the messages are delivered using Store-CarryForward routing by which the nodes can exchange data whenever they come in close. If there is no direct connection from source to destination, data holding nodes will discover their nearest neighbor nodes to forward messages toward the destination node as shown in Fig. 1. There are several existing works in the literature [6]-[12] with the aim for $100 \%$ delivery ratio which is quite difficult to achieve especially in sparse 
networks with constraints in energy consumption and message delivery deadline.

Vahdat et al. [6] proposed the epidemic routing using the uncontrolled flooding algorithm in which the replication of source data is not restricted with any limits in order to route the message from source to destination in the intermittently connected network. However, this type of routing incurs a significant demand on both bandwidth and buffer capacity. To address the excess traffic overhead, Khaled et al. [7] proposed a Controlled Flooding scheme which can limit the flooding by three parameters: Willingness probability, Time-to-Live, and Kill Time. Nevertheless, flooding based routing performance degradation has been reported in a very sparse network [8].

Lindgren et al. [9] proposed a prediction based routing called PROPHET (Probabilistic Routing Protocol using History of Encounters and Transitivity) by estimating the delivery predictability to indicate the probability of success in delivering a message to the destination from the local node. In this prediction based routing category, Brun et al. [10] also proposed a protocol utilizing the motion vector of mobile nodes to predict the future location of mobile nodes by using the knowledge of relative velocities of a node and its neighbor nodes to predict the closest distance between two nodes. Although the prediction based approach can reduce traffic overhead in the network, but it fails to improve the performance in an extremely low node density scenario and, in some cases, results in the delivery ratio reduction.

To refine the prediction based routing, Boldrini et al. [11] proposed the History based routing (HiBOp) which exploits current context information for data forwarding decisions. Even though this context based routing approach can reduce the resource consumption in terms of network traffic and storage, its delay performance is significantly inferior to that of the Epidemic algorithm.

Commonly, OppNets can be deployed on several scenarios such as distributed vehicular data sensing where the usergenerated data are gathering from crowd-sourcing users and service providers. The existing example of this scenario is Waze [13] which utilizes the floating car data (FCD)from positioning data such as GPS localization, information about cellular hand-offs of mobile devices, and coverage of WiFi networks to improve real-time traffic estimates. Similar to previous scenario, smart cities data sensing is also using mobile devices to collect the sensing data in addition to the position data such as camera, microphone, accelerometer or gyroscope. In addition to that, step counters, movement, pressure, temperature and humidity sensors are added to the new features of the common smart phones nowadays. Another popular scenario is opportunistic social networks where the delay tolerant data are exchanged base on the groups formed by the users mutual'interests. The examples of this case are Opportunistic computing [14], Recommender systems [15] and Mobile data offloading such as MoSoNets [16]. Overall, the performance of most existing algorithms are degrading in very sparse node density, and the energy consumption is not taken into the consideration which is a crucial factor in mobile sensor devices.

Undoubtedly, an energy efficient forwarding mechanisms is a must for OppNets since most nodes are usually mobile de- vices with limited battery capacity. Martin et al. [17] proposes PropTTR and PropNTTR, a set of energy-efficient forwarding mechanisms based on MaxProp [18] for emergency scenarios using wireless OppNets for communication between nodes. Since in emergency scenarios, it is crucial to preserve battery while maintaining the message deliverable. Both PropTTR and PropNTTR demonstrate an acceptable delivery ratio with a low energy consumption. Chen et al. [19] propose assistant opportunistic routing (AsOR) protocol which is an efficient and energy conservative unicast routing technique for multihop wireless sensor networks over Rayleigh fading channels. Numerical results show that this protocol is energy conservative compared with other traditional routing protocols. Perales et al. [20] demonstrates that significant energy-efficiency gains can be achieved when Multihop Cellular Networks (MCNs) jointly exploit opportunistic networking and the traffic delaytolerance characteristics of relevant mobile data services.

Nevertheless, most of energy conservative protocols proposed for opportunistic routing evaluation are unaware of the mobility model used in the scenarios. There are two random processes given by mobility that underlie opportunistic communication:

- the opportunity for contacts among nodes.

- the opportunity for meetings between specific nodes.

Our aim for this research is to study the impact of mobility model on the energy consumption of the mobile nodes in the OppNets.

\section{EVALUATION}

\section{A. Metrics}

1) Mobility model: We compare 5 common mobility models. The most traditional models are Random Walk (RW), Random Waypoint (RWP) and Random Direction (RD). These models are based on uniform distributions for userdefined direction, speed and pause times.

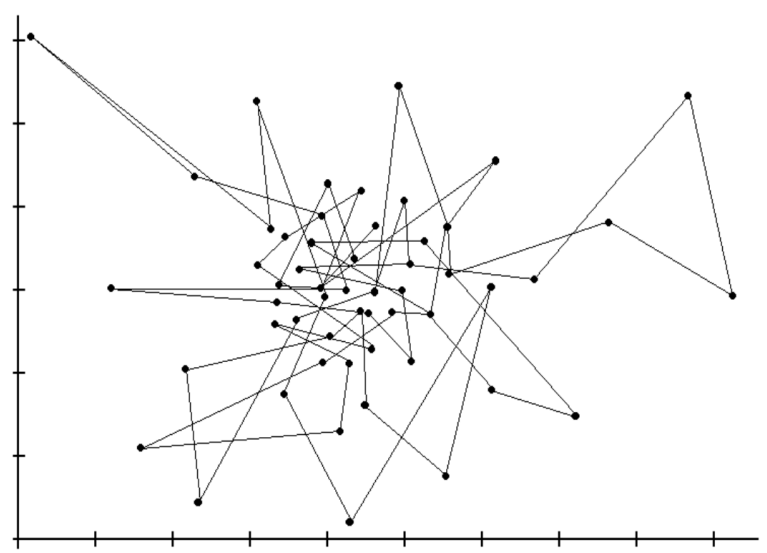

Fig. 2. Random Walk model mobility movement pattern [21]

In RW, The speed of a node is randomly selected and its direction is also randomly chosen. Then, each node goes in the selected random direction with the selected speed until the epoch lasts. Each epoch's duration is again randomly selected [22] as in Fig. 2. This mobility model was created 
with the purpose to mimic the natural extremely unpredictable movement of people. A mobile node moves from its current location to a new location by randomly choosing a direction and speed from pre-defined ranges to travel. For every new interval $t$, each node randomly and uniformly chooses its new direction $\theta(t)$ from $[0,2 \pi]$. Similarly, the new speed $v(t)$ follows a uniform distribution or a Gaussian distribution from $\left[0, V_{\max }\right]$. Thus, during time interval $t$, the node moves with the velocity vector:

$$
[v(t) \cos \theta(t), v(t) \sin \theta(t)]
$$

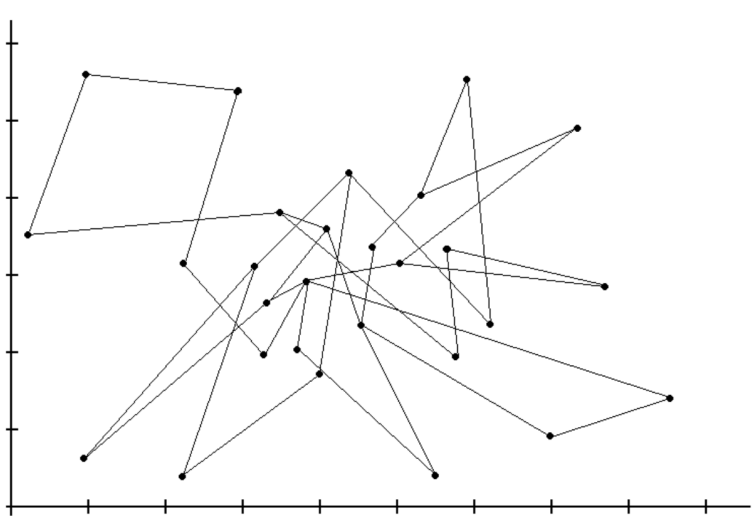

Fig. 3. Random Waypoint mobility movement pattern [21]

In RWP model, a new destination inside the network area is chosen randomly. Then the node moves towards that destination with a randomly selected speed as in Fig. 3. Normally the Network Simulator implements this mobility model as follows:

- A node randomly chooses a destination and moves towards it with a velocity chosen uniformly and randomly from pre-defined ranges $[0, V \max ]$

- The direction and velocity of a node are chosen independently of other nodes.

- Upon reaching the destination, the node stops for the pause time parameter $T_{\text {pause }}$ duration.

- After this duration, it again chooses a random destination and repeats the whole process again until the simulation ends.

In RD model, a mobile node makes random mobility decisions with respect to current time or location, independent of other nodes. A node randomly picks a movement direction, and takes straight-line movement towards that direction for a given distance [23]. In this mobility model, the mobile node chooses any random direction to travel until the boundary of edge is found.

The simplest map-based mobility model is Random MapBased Movement (MBM). Nodes adopting this model move randomly but always in the streets described on the map.

This Shortest Path Map-Based Movement (SPMBM) is another mobility model that uses a map-described environment to restrict node movement. With this model, nodes choose their destination randomly inside the map, then calculate the shortest

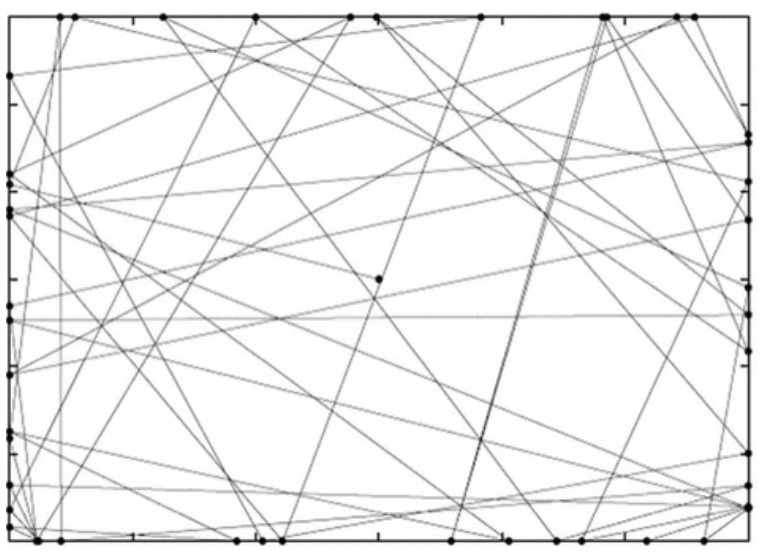

Fig. 4. Random Direction mobility movement pattern [24]

path to reach it and finally walk along this path. We use the Helsinki map as a based provide by Opportunistic Networking Environment (ONE) [25] simulator which specifically designed for evaluating OppNets routing and application protocols. To sum up, we conclude the properties of evaluated mobility models in Table I.

TABLE I. SUMMARIZE OF MOBILITY PROPERTIES

\begin{tabular}{llll}
\hline Mobility model & Direction & Velocity & Stop and obstacles \\
\hline Random Walk & random & random & no \\
Random Waypoint & random & random & no \\
Random Direction & random & random & border \\
Shortest Path Map Based & street map based & variable & no \\
Random Map Based & street map based & variable & \\
\hline
\end{tabular}

2) Energy model: Normally the power consumption on the OppNets can be computed as power loss model as [4]:

$$
E_{T}=L \cdot \epsilon_{f_{s}} \cdot d^{\alpha}
$$

where $E_{T}$ is an amount of energy consumed at a node for transmitting $L$ length message. In the equation, $\alpha$ is the power loss component with $\alpha \in[2,4]$. Additionally, $\epsilon_{f_{s}}[\mathrm{~J} /(\mathrm{bit} / \mathrm{m})]$ is the amount of energy consumed by an amplifier to transmit one bit data at an acceptable quality level.

Our implementation of energy model assumes that each node has five states:

- Transmission: In this state, a node is transmitting messages.

- Reception: In this state, a node is receiving messages.

- Scan: Each node is usually change to this state based on the scanInterval time in order to detect the neighbor nodes.

- Inactive: The network interface is sleeping in this state but it can be discovered by other nodes.

- Off: The network interface is turn offed during this state and cannot establish any connection.

For the energy configuration settings, we set up the parameters as in Table II. 
TABLE II. ENERGY CONFIGURATIONS

\begin{tabular}{ll}
\hline Parameters & Configurations \\
\hline Battery Capacity & 4.8 Joules \\
Scanning & $0.92 \mathrm{~mW} / \mathrm{s}$ \\
Transmitting & $0.08 \mathrm{~mW} / \mathrm{s}$ \\
Receiving & $0.08 \mathrm{~mW} / \mathrm{s}$ \\
\hline
\end{tabular}

TABLE III. PARAMETER CONFIGURATION

\begin{tabular}{ll}
\hline Parameters & Settings \\
\hline Simulation Time & $5000 \mathrm{~s}$. \\
Number of Nodes & 50 \\
Router & Epidemic router \\
Message Size & $500 \mathrm{~KB}-1 \mathrm{MB}$ \\
Message generated rate & 1 per $25-35 \mathrm{~s}$. \\
Node Buffer & $5 \mathrm{MB}$ \\
Transmission Speed & $250 \mathrm{k}$. \\
Radio Range & $10 \mathrm{~m}$. \\
Moving Speed & $0.5-1.5 \mathrm{~m} / \mathrm{s}$ \\
\hline
\end{tabular}

3) Results: We compare the energy consumption of the nodes with five mobility models varying by the number of node in the area. The result from Fig. 5 presents the average power usage per node on the node density. This graph shows that the MapBased movement model utilize more power than the stochastic routing models while shortest path map base movement model consumes the most power. In the random movement model, all three random based movement models consume similar amount of power while RD utilize more energy than RWP and RW respectively. In all cases, the energy consumption increases with the number of nodes.

In addition, we analyze the relationship between the delivery ratio and energy consumption as in Fig. 6. This graph shows the ratio of delivery probability of each mobility model and the energy usage. The result illustrates that the SPMB and MBM outperform the random based movements. This is a predictable outcome since MapBase routing can gain much higher delivery ratio than the random based movement. In the stochastic mobility model, RD performs better than RWP and RD respectively. This is the results from energy utilization since RWP and RD contains more stops and changes in direction than RD counterpart.

In Fig. 7, the relation of average latency and energy consumption on node density is displayed. This graph show that RW gain less average latency and energy consumption ratio than other model which is presenting a good performance with power utilization. However, on the higher node density, MBM perform better when the number of node are over 30 nodes. Note that, the ratio of average latency and energy consumption of RWP tends to be stable when the amount of node are over 20 nodes.

4) Discussions: The results from the simulations show the energy utilization of each mobility model widely used in the OppNet simulation. The random based mobility models clearly present the lower energy consumption comparing to the map based mobility models. In the case of random based movement models, the graph in Fig. 5 show that RD use more energy than RWP and RW respectively while node density has no impact on the power consumption. Comparing to the mobility movement patterns in Fig. 2, 3 and 4, we can observe that mobility with less stops (in order to change direction) such as RD in Fig. 4 tends to use more energy than RWP and

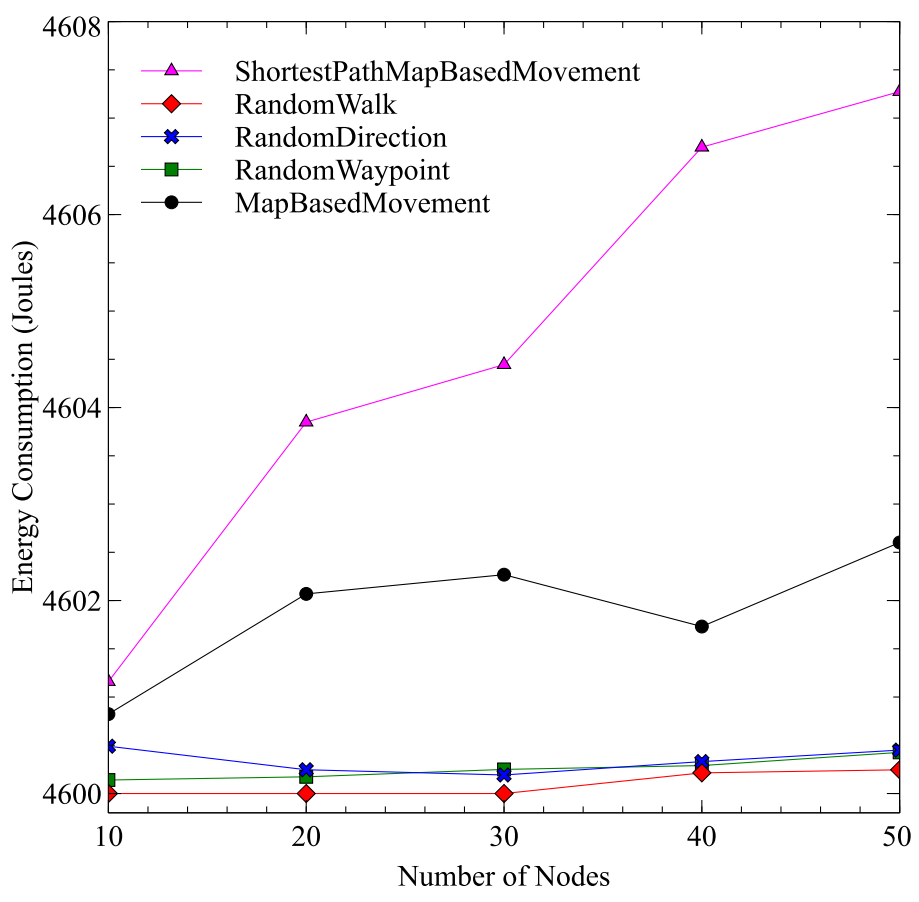

Fig. 5. The Average Energy Consumption on Movement Models

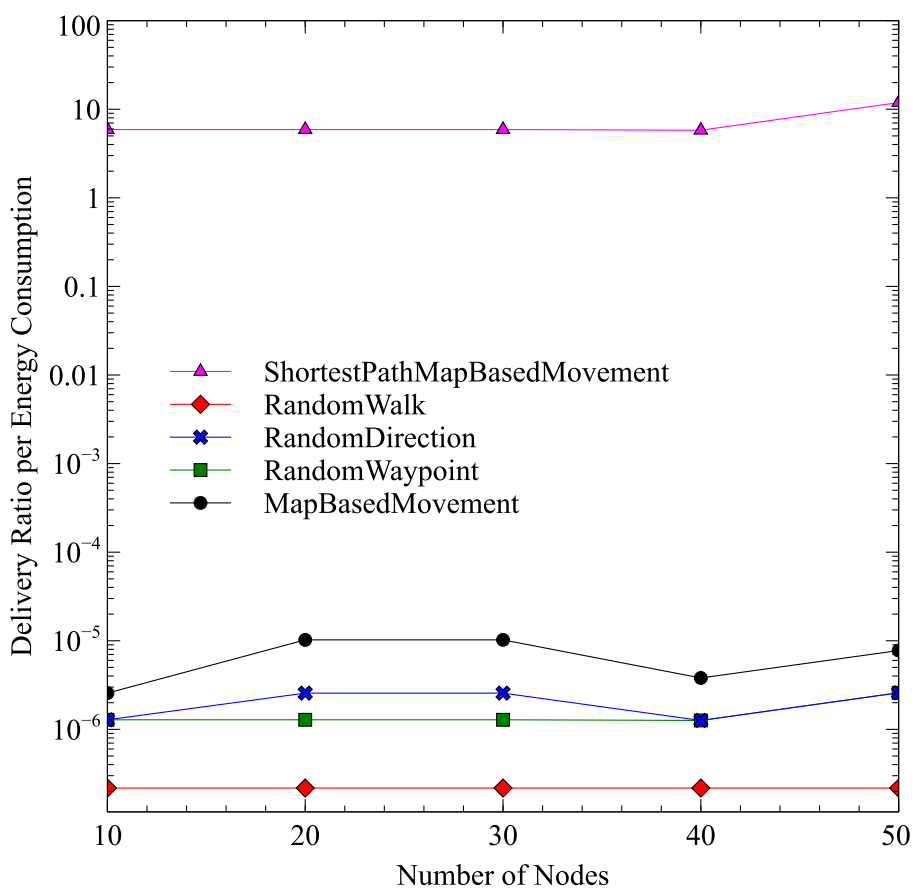

Fig. 6. Delivery Ratio per Energy Consumption

RW in Fig. 3 and Fig. 2 respectively. Therefore, we can see the reverse variation relationship of energy consumption and number of the stops in the mobility pattern. The aim of this study is to observe mainly on the random based mobility model, although we compare with map based mobility model in order to complete the analytical process. Nevertheless, this is the preliminary results from our observation. The extensive studies to clarify this result is required to be extended. After 


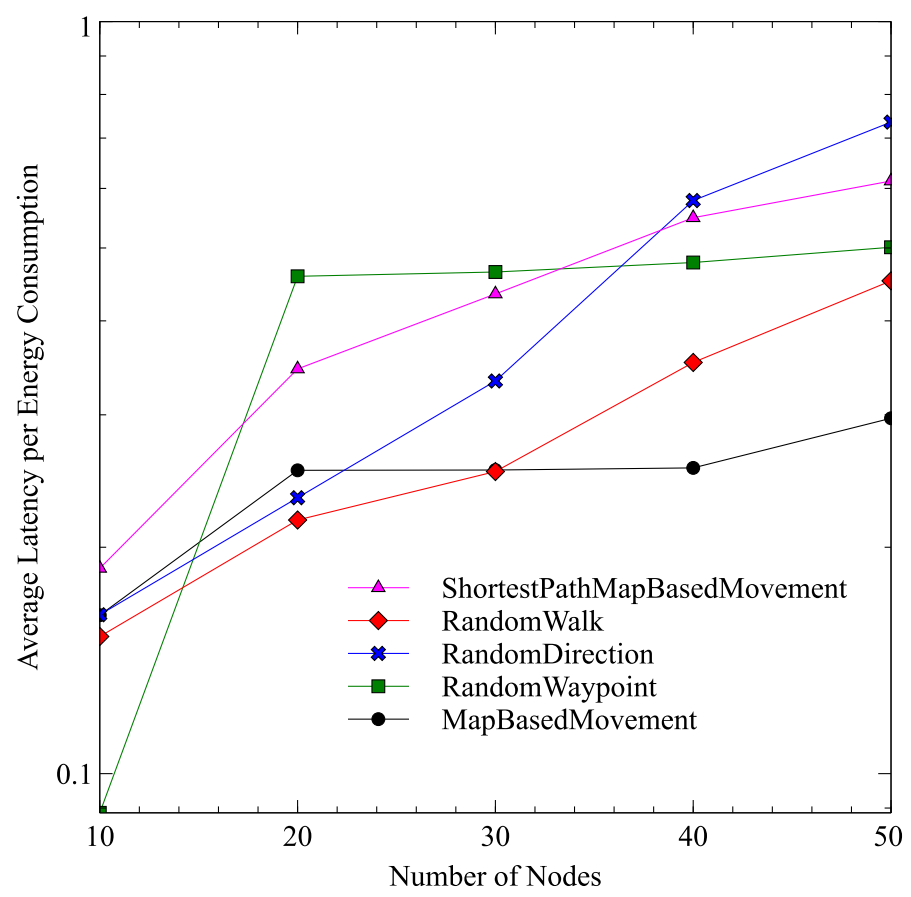

Fig. 7. Average Latency per Energy Consumption

our extensive simulations, we found that the mobility models designed with map and without map (random based) should be separately evaluated in order to make more efficiency analysis. On the random based routing, we found the parameters of each model that affects the performance of opportunistic routing algorithm are moving pattern and number of stops. From our observation, the delivery ratio of the models tend to be lower if the nodes frequently change their direction. This means if the nodes make more stops, they prone to meet less contacted nodes which can affect the performance of OppNet routing. In addition, most researchers commonly choose mobility model based on the actual behavior that appropriate to their designed scenario. The significant of our study is to find the relationship of the parameter in OppNet routing that affect the power consumption in the OppNet environments.

\section{CONCLUSiON}

This paper study the affect of OppNet mobility models on the energy consumption since the power utilization is a critical factor in the portable devices in the OppNet scenarios. We compare five mobility models: Random Walk, Random Waypoint, Random Direction, Random Map-Based Movement and Shortest Path Map-Based Movement. The results from the simulations show that random direction consume more power than random waypoint and random walk respectively. In the MapBased mobility models, Shortest Path Map-Based Movement consume more energy than Random Map-Based Movement while gaining higher delivery ratio. To extend this work in the future, we can increase the scenarios used in the simulation, in order to gather more data to validate the results with in-depth extensive simulations.

\section{ACKNOWLEDGMENT}

The author would like to thanks the support from Basic Research Program at Data Communication Laboratory, Defence Technology Institute, Thailand.

\section{REFERENCES}

[1] L.-J. Chen, C.-H. Yu, C.-L. Tseng, H.-h. Chu, and C.-F. Chou, "A content-centric framework for effective data dissemination in opportunistic networks," IEEE Journal on Selected Areas in Communications, vol. 26, no. 5, pp. 761-772, Jun. 2008.

[2] V. F. Mota, F. D. Cunha, D. F. Macedo, J. M. Nogueira, and A. A. Loureiro, "Protocols, mobility models and tools in opportunistic networks: A survey," Computer Communications, vol. 48, no. 0, pp. 5 19, 2014, opportunistic networks.

[3] L. Pajevic, "Opportunistic Networking: Mobility Modeling and Content Distribution," Ph.D. dissertation, KTH, Stockholm, Sweden, 2013.

[4] W. Yang, W. Liang, and W. Dou, "Energy-Aware Real-Time Opportunistic Routing for Wireless Ad Hoc Networks," 2010 IEEE Global Telecommunications Conference GLOBECOM 2010, pp. 1-6, Dec. 2010.

[5] J. Kerdsri and K. Wipusitwarakun, "Dynamic Rendezvous Based Routing Algorithm on Sparse Opportunistic Network Environment," International Journal of Distributed Sensor Networks, vol. 2015, pp. $1-12,2015$.

[6] A. Vahdat and D. Becker, "Epidemic Routing for Partially-Connected Ad Hoc Networks," Technical Report CS-200006, Duke University, Tech. Rep.

[7] K. A. Harras, K. C. Almeroth, and E. M. Belding-Royer, "Delay Tolerant Mobile Networks (DTMNs): Controlled Flooding in Sparse Mobile Networks," in Proceedings of the 4th IFIP-TC6 International Conference on Networking Technologies, Services, and Protocols, ser. NETWORKING'05. Berlin, Heidelberg: Springer-Verlag, 2005, pp. $1180-1192$.

[8] V. V. Neena and V. M. A. Rajam, "Performance analysis of epidemic routing protocol for opportunistic networks in different mobility patterns," 2013 International Conference on Computer Communication and Informatics, pp. 1-5, Jan. 2013.

[9] A. Lindgren, A. Doria, and O. Schelén, "Probabilistic routing in intermittently connected networks," ACM SIGMOBILE Mobile Computing and Communications Review, vol. 7, no. 3, p. 19, Jul. 2003.

[10] B. Burns, O. Brock, and B. N. Levine, "Mv routing and capacity building in disruption tolerant networks," in In Proc. IEEE INFOCOM, 2005, pp. 398-408.

[11] C. Boldrini, M. Conti, J. Jacopini, and A. Passarella, "Hibop: a history based routing protocol for opportunistic networks," in World of Wireless, Mobile and Multimedia Networks, 2007. WoWMoM 2007. IEEE International Symposium on a, June 2007, pp. 1-12.

[12] J. Kerdsri and K. Wipusitwarkun, "DORSI : Data-wise Opportunistic Routing with Spatial Information," Journal of Convergence Information Technology, vol. 8, no. August, pp. 91-103, 2013.

[13] T. . N. App. (2015) Free community-based mapping. [Online]. Available: http://www.waze.com

[14] M. Conti, S. Giordano, M. May, and A. Passarella, "From opportunistic networks to opportunistic computing," Communications Magazine, IEEE, vol. 48, no. 9, pp. 126-139, Sept 2010.

[15] C. Tekin, S. Zhang, and M. van der Schaar, "Distributed online learning in social recommender systems," Selected Topics in Signal Processing, IEEE Journal of, vol. 8, no. 4, pp. 638-652, Aug 2014.

[16] B. Han, P. Hui, V. S. A. Kumar, M. V. Marathe, J. Shao, and A. Srinivasan, "Mobile data offloading through opportunistic communications and social participation," IEEE Transactions on Mobile Computing, vol. 11, no. 5, pp. 821-834, May 2012. [Online]. Available: http://dx.doi.org/10.1109/TMC.2011.101

[17] A. Martín-Campillo and R. Martí, "Energy-efficient forwarding mechanism for wireless opportunistic networks in emergency scenarios," Computer Communications, vol. 35, no. 14, pp. 1715-1724, Aug. 2012. 
[18] J. Burgess, B. Gallagher, D. Jensen, and B. Levine, "Maxprop: Routing for vehicle-based disruption-tolerant networks," in INFOCOM 2006. 25th IEEE International Conference on Computer Communications. Proceedings, April 2006, pp. 1-11.

[19] C. Wei, C. Zhi, P. Fan, and K. Ben Letaief, "Asor: an energy efficient multi-hop opportunistic routing protocol for wireless sensor networks over rayleigh fading channels," Wireless Communications, IEEE Transactions on, vol. 8, no. 5, pp. 2452-2463, May 2009.

[20] B. Coll-Perales, J. Gozalvez, O. Lazaro, and M. Sepulcre, "Opportunistic multihopping for energy efficiency: Opportunistic multihop cellular networking for energy-efficient provision of mobile delaytolerant services," Vehicular Technology Magazine, IEEE, vol. 10, no. 2 , pp. 93-101, June 2015.

[21] A. M. de Almeida e Silva, "Simulation of telecommunicati on networks for public transportation systems," 2008.

[22] E. Bulut, "OPPORTUNISTIC ROUTING ALGORITHMS," Ph.D. dissertation, Rensselaer Polytechnic Institute, 2011.

[23] G. S. Thakur, U. Kumar, A. Helmy, and W.-J. Hsu, "On the efficacy of mobility modeling for DTN evaluation: Analysis of encounter statistics and spatio-temporal preferences," 2011 7th International Wireless Communications and Mobile Computing Conference, pp. 510-515, Jul. 2011.

[24] R. Roy, "Realistic random direction mobility," in Handbook of Mobile Ad Hoc Networks for Mobility Models. Springer US, 2011, pp. 223244.

[25] A. Keränen, J. Ott, and T. Kärkkäinen, "The ONE simulator for DTN protocol evaluation," Proceedings of the Second International ICST Conference on Simulation Tools and Techniques, 2009. 